\title{
Cytoglobin/STAP, its unique localization in splanchnic fibroblast-like cells and function in organ fibrogenesis
}

\author{
Kazuki Nakatani ${ }^{1, \dagger}$, Hiroaki Okuyama ${ }^{2, \dagger}$, Yasuyuki Shimahara ${ }^{2}$, Shigeru Saeki ${ }^{3}$, Dong-Ho \\ $\mathrm{Kim}^{3}$, Yuji Nakajima ${ }^{1}$, Shuichi Seki ${ }^{4}$, Norifumi Kawada ${ }^{4}$ and Katsutoshi Yoshizato ${ }^{5,6,7}$ \\ ${ }^{1}$ Department of Anatomy, Graduate School of Medicine, Osaka City University, Japan; ${ }^{2}$ Department of \\ Gastroenterological Surgery, Graduate School of Medicine, Kyoto University, Japan; ${ }^{3}$ Department of Human \\ Life Science, Osaka City University, Japan; ${ }^{4}$ Department of Hepatology, Graduate School of Medicine, Osaka \\ City University, Japan; ${ }^{5}$ Hiroshima Tissue Regeneration Project, Hiroshima Prefecture Collaboration of \\ Regional Entities for the Advancement of Technological Excellence, JST, Japan; ${ }^{6}$ Cooperative Link of Unique \\ Science and Technology for Economy Revitalization and ${ }^{7}$ Developmental Biology Laboratory, Department of \\ Biological Science, Graduate School of Science, Hiroshima University, Hiroshima, Japan
}

\begin{abstract}
Cytoglobin/stellate cell activation-associated protein (Cygb/STAP) consists of a new class of hexacoordinate globin superfamily, which was recently discovered by a proteome analysis on the rat hepatic stellate cells. Unlike haemoglobin, myoglobin, and neuroglobin, Cygb/STAP is ubiquitously expressed in several organs, although its detailed localization has not been clarified. Immunohistochemistry and immunoelectron microscopy revealed that Cygb/STAP is uniquely localized in fibroblast-like cells in splanchnic organs, namely the vitamin A-storing cell lineage, but neither in epithelial cells, endothelial cells, muscle cells, blood cells, macrophages, nor dermal fibroblasts. The expression of Cygb/STAP was upregulated in fibrotic lesions of the pancreas and kidney in which activated fibroblast-like cells or myofibroblasts are known to increase in number. In cultured hepatic stellate cells, Cygb/STAP expression was augmented by the stimulation with sera, plateletderived growth factor-BB, and transforming growth factor- $\beta$ 1. Overexpression of Cygb/STAP in NIH 3T3 cells induced the cells to lessen migratory activities and increase the expression of collagen $\alpha 1$ (I) mRNA. These results indicate that Cygb/STAP is a tissue globin uniquely localized in splanchnic fibroblastic cell lineage and may play a role in fibrotic organ disorder.

Laboratory Investigation (2004) 84, 91-101, advance online publication, 1 December 2003; doi:10.1038/labinvest.3700013
\end{abstract}

Keywords: stellate cells; fibroblasts; vitamin A; collagen $\alpha 1(\mathrm{I})$; PDGF; TGF $\beta$; NIH 3 T3 cells

Stellate cell activation-associated protein (STAP) is a haemoprotein that was recently discovered by a proteome approach for the molecular analysis of the activation of rat hepatic stellate cells (HSCs), liverspecific fibroblast-like cells storing vitamin A. ${ }^{1}$ Homologous proteins were successively cloned as mouse and human genes, and named cytoglobin $(\mathrm{Cygb})^{2,3}$ or histoglobin. ${ }^{4}$ These proteins as well as neuroglobin (Ngb) consist of hexacoordinate globin superfamily and have $\mathrm{O}_{2}$-binding capacity compar-

Correspondence: Katsutoshi Yoshizato, Developmental Biology Laboratory, Department of Biological Science, Graduate School of Science, Hiroshima University, 1-3-1, Kagamiyama, Higashihiroshima, Hiroshima 739-8526, Japan.

E-mail: kyoshiz@hiroshima-u.ac.jp

KN and HO equally contributed to this study.

Received 21 July 2003; revised 24 September 2003; accepted 26 September 2003; published online 1 December 2003 able to that of myoglobin. Their principal role is speculated to supply $\mathrm{O}_{2}$ to mitochondria, although no information on its function has been available to date. $^{3,4}$ Taking the general distribution and molecular structures of STAP into consideration, we rename STAP as Cygb/STAP in the present study.

In the rat liver, Cygb/STAP is localized in the quiescent HSCs and is dramatically increased when these cells are activated in fibrotic liver tissues. ${ }^{1}$ As well characterized, the activated HSCs show a myofibroblastic phenotype. ${ }^{5}$ Other hepatic constituent cells in liver lobules such as Kupffer cells (macrophages), endothelial cells, hepatocytes, and bile-duct epithelial cells are negative for Cygb/STAP immunostaining. ${ }^{1}$ Cygb/STAP-positive cells in the human liver are distributed as in rat. ${ }^{6}$

HSCs are vitamin A-storage cells in the liver. Vitamin A-containing fibroblast-like cells are also present in the parenchyma of various extrahepatic 
organs, although not so abundant as compared to those in HSCs. ${ }^{7-9}$ These splanchnic fibroblast-like cells are thought to be closely related to HSCs. ${ }^{8}$ However, this speculation has not been experimentally verified. We thought that Cygb/STAP is a useful molecular marker to test the possible relationship between the extrahepatic vitamin A-containing cells and HSCs. There have been studies on the expression of Cygb/STAP mRNAs in various mouse and human organs. ${ }^{2-4,6}$ However, Cygb/STAP-positive cells in these organs have not been characterized well and identified. We propose here that Cygb/STAP represents a novel marker for the splanchnic fibroblasts that are in vitamin A-storing cell lineage, and show that Cygb/STAP is induced by chronic tissue damage accompanying fibrosis. In addition, the present study demonstrates that Cygb/STAP regulates the migration and the collagen gene expression of fibroblasts and, thus, suggests an important role of Cygb/STAP in the fibrogenic process.

\section{Materials and methods}

\section{Animals}

Male Wistar and Sprague-Dawley (SD) rats were purchased from Charles River Japan (Kanagawa, Japan). Male Wistar Bonn/Kobori (WBN/Kob) rats were provided by Japan SLC (Shizuoka, Japan). These animals were all specific pathogen free. Experiments were conducted in accordance with the guideline for animal experiments of the Osaka City University Graduate School of Medicine.

\section{Induction of Experimental Chronic Nephropathy}

Six SD rats fed a low-salt diet $(0.05 \%$ sodium, Oriental Yeast, Osaka, Japan) were administered subcutaneously every day for 28 days with $15 \mathrm{mg} / \mathrm{kg}$ body weight of cyclosporin A (Wako Pure Chemical Co., Osaka, Japan) diluted in olive oil. ${ }^{10}$

\section{Preparation of HSCs}

HSCs were isolated from rat livers as described previously. ${ }^{1}$ HSCs were plated and cultured on noncoated plastic plates (FALCON 3001) in Dulbecco's modified Eagle's medium (DMEM, Gibco BRL, Gaithersburg, MD, USA) supplemented with or without $10 \%$ fetal bovine serum (FBS, Gibco BRL). The culture medium was changed every other day unless otherwise mentioned. In some experiments, HSCs were treated with recombinant rat platelet-derived growth factor (PDGF)-BB, recombinant human transforming growth factor-beta 1 (TGF- $\beta 1$, R\&D system, Minneapolis, MN, USA), PD98059, LY294002, and GF109203X (Calbiochem, La Jolla, CA, USA).

\section{Antibodies}

Rabbit polyclonal IgG antibodies against rat Cygb/ STAP were produced by using a synthetic $\mathrm{NH}_{2}$ - terminal polypeptide of rat Cygb/STAP, $\mathrm{NH}_{2}$ MEKVPGDMEIERRERNEE + Cys-COOH, as an immunogen as described previously. ${ }^{1}$ Mouse monoclonal IgG1 antibodies against rat Thy-1, CD73, ED1like antigen (ED1), and OX62 mouse monoclonal IgG1 antibodies, which recognize the rat homologue of integrin alpha E2, ${ }^{11,12}$ were purchased from Phar Mingen (San Diego, CA, USA). Mouse monoclonal IgG2b antibodies against rat heat-shock protein (HSP) 47 were provided by Calbiochem-Novabiochem Co. (San Diego, CA, USA). Mouse monoclonal IgG2a antibodies against human alpha smooth muscle actin ( $\alpha \mathrm{SMA})$ that react to rat $\alpha \mathrm{SMA}^{13}$ were provided by DAKO (Glostrup, Denmark). Rabbit polyclonal IgG antibodies against human c-kit that react to rat $\mathrm{c}^{-\mathrm{kit}^{14}}$ were purchased from Oncogene Research Products (Boston, MA, USA). Rabbit polyclonal IgG antibodies against human plateletderived growth factor receptor-beta (PDGFR- $\beta$ ) that react to rat PDGFR- $\beta,{ }^{1}$ and rabbit polyclonal IgG antibodies against rat vinculin were purchased from Santa Cruz Biotechnology (Santa Cruz, CA, USA). For negative controls, we used mouse monoclonal antibodies against IgG1, IgG2a, and IgG2b (DAKO) or rabbit polyclonal IgG antibodies (Santa Cruz Biotechnology), all of which do not recognize rat proteins. For the secondary antibodies, we used horse radish peroxidase (HRP)-conjugated rabbit polyclonal anti-mouse IgG antibodies, HRP-conjugated swine polyclonal anti-rabbit IgG antibodies, FITC-labelled goat polyclonal anti-rabbit IgG antibodies, biotinylated donkey polyclonal anti-mouse and rabbit IgG antibodies in an LSAB-2 kit (DAKO), HRP-conjugated goat polyclonal anti-mouse and rabbit IgG antibodies, named as Histofine Simple Stain Rat MAX-PO (Nichirei, Tokyo, Japan), Alexa Fluor 488 goat anti-rabbit IgG $(\mathrm{H}+\mathrm{L})$ antibodies, and Alexa Fluor 594 goat anti-mouse IgG $(\mathrm{H}+\mathrm{L})$ antibodies (Molecular Probes, Eugene, OR, USA).

\section{Western Blot}

Protein samples (10 $\mu \mathrm{g}$ of protein) were subjected to SDS-PAGE and electrically transferred onto Immobilon P membranes (Millipore Corp., Bedford, MA, USA). The membranes were blocked with $5 \%$ skim milk, treated with primary antibodies, and were incubated with peroxidase-conjugated secondary antibodies. Immunoreactive bands were visualized by using the ECL system (Amersham). The density of each band was analysed by Imaging Densitometer GS-700 (BIO-RAD, Hercules, CA, USA).

\section{Immunohistochemistry and Immunoelectron Microscopy}

Immunohistochemistry was performed by methods described elsewhere. ${ }^{15,16}$ For immuno-light microscopy, $4 \%$ paraformaldehyde-fixed and frozen specimens were cut into $5-\mu \mathrm{m}$-thick sections. After blocking with $5 \%$ bovine serum albumin (BSA)/ 
phosphate-buffered saline (PBS), they were incubated overnight with primary antibodies in 5\% BSA/PBS. Endogenous peroxidase was then blocked by incubating sections in methanol containing $0.3 \%$ $\mathrm{H}_{2} \mathrm{O}_{2}$. In the subsequent reaction, we used Histofine Simple Stain Rat MAX-PO as secondary antibodies. Immunoreaction was visualized by treating with $0.25 \mu \mathrm{g} / \mathrm{ml} \quad 3,3^{\prime}$-diaminobenzidine tetrahydrochloride (Wako Pure Chemical Co.) and 0.003\% $\mathrm{H}_{2} \mathrm{O}_{2}$ in $0.05 \mathrm{M}$ Tris buffer. Specimens were counterstained for nuclei with 5\% methyl green (Muto Pure Chemicals, Tokyo, Japan). Immunoelectron microscopic analysis was performed on $50-\mu \mathrm{m}$-thick sections of the kidney and spleen, and $5-\mu \mathrm{m}$-thick sections of the pancreas, small intestine, and lung. In advance, endogenous biotin on specimens was blocked by a Biotin Blocking system (DAKO). They were stained for Cygb/STAP using an LSAB-2 kit in a similar way as described previously, ${ }^{13,16,17}$ postfixed in 1\% osmium tetraoxide (Merck), dehydrated in ethanol, and embedded in Polybed (Polyscience Inc., Warrington, PA, USA). Ultrathin sections were observed under a JEM-1200 EX electron microscope (JEOL, Tokyo, Japan) at $100 \mathrm{kV}$.

\section{Double Immunostaining}

Double immuno-staining analysis was carried out by methods described previously. ${ }^{18}$ Frozen specimens were fixed with $4 \%$ paraformaldehyde, cut into $5 \mu \mathrm{m}$-thick sections, blocked with $5 \%$ BSA/PBS, and were incubated overnight with primary antibodies. They were then incubated with $20 \mu \mathrm{g} / \mathrm{ml}$ Alexa Fluor 488 goat anti-rabbit antibodies and $20 \mu \mathrm{g} / \mathrm{ml}$ Alexa Fluor 594 goat anti-mouse antibodies for $2 \mathrm{~h}$. The sections were observed under an LSM510 confocal laser scanning microscope (Carl Zeiss, Germany).

\section{Generation of Rat Cygb/STAP-expressing NIH 3T3 Cell Lines}

Rat Cygb/STAP cDNA was cloned into pBluescript SK( $(-)$ as previously described. ${ }^{1}$ The cDNA fragment was ligated into pZeoSV2(+ ) (Invitrogen, Carlsbad, CA, USA) at an EcoRI restriction site. A control vector that included lacZ instead of Cygb/STAP cDNA was also prepared. These vectors were transfected into subconfluent NIH3T3 cells using Lipofectamine 2000 (Invitrogen, Carlsbad, CA, USA) in OPTI-MEM medium (Invitrogen). The cells were grown in FBS/DMEM and successively selected by $200 \mu \mathrm{g} / \mathrm{ml}$ Zeocin (Invitrogen). Cell clones were obtained by using a limited dilution method. Cygb/ STAP expression was checked by Western blotting and by indirect immunofluorescence against rat Cygb/STAP. Among Cygb/STAP-expressing cell lines obtained, a cell line named F9 was used in the present study.

\section{Staining of F-actin and Vinculin}

Cultured NIH3T3 cells were fixed with $3.7 \%$ formaldehyde for $30 \mathrm{~min}$ at room temperature. After washing three times with PBS containing $0.1 \%$ Triton X-100, the cell-associated F-actin was stained by $0.5 \mu \mathrm{M}$ TRITC-phalloidin (Sigma) for $1 \mathrm{~h}$ at room temperature. For the detection of vinculin, the fixed cells were incubated with polyclonal anti-vinculin antibodies for $1 \mathrm{~h}$ at room temperature and successively with FITC-labelled goat anti-rabbit IgG for $1 \mathrm{~h}$ at room temperature. After washing, the specimens were observed under a fluorescent microscope.

\section{Migration Assay}

The migration activity of NIH 3T3 cells was assayed by using Cell Culture Insert as described previously. ${ }^{19} \mathrm{NIH}$ 3T3 cells $\left(1 \times 10^{5}\right.$ cells $)$ were detached from the plates using trypsin, suspended in $400 \mu \mathrm{l}$ of $10 \% \mathrm{FBS} / \mathrm{DMEM}$, introduced into the insert (pore size of $8 \mu \mathrm{m}$, FALCON 3093), and allowed to adhere to the upper surface of the membrane. The cells were then maintained in $10 \% \mathrm{FBS} / \mathrm{DMEM}$ for $24 \mathrm{~h}$. After $48 \mathrm{~h}$ of incubation, the culture medium was removed. Cells adhering to the membrane were fixed by $100 \%$ methanol and stained with a Giemsa solution. The number of cells on the upper surface of the membrane was counted at a $\times 400$ magnification and similarly for the lower surface by changing the focus. Five microscopic fields were randomly chosen for each specimen. The rate (\%) of migrated cells, migration index (MI), was calculated by dividing the number of the cells on the lower surface of the membrane by the number of upper and lower surfaces of the membrane.

\section{Northern Blot}

Total RNA was extracted from NIH3T3 cells using Isogen (Nippon Gene, Tokyo, Japan). Total RNA $(10 \mu \mathrm{g})$ was separated on a $1 \%$ agarose gel, and transferred onto nylon membranes. After prehybridization, membranes were incubated in buffer supplemented with PCR-amplified double-stranded mouse collagen $\alpha 1$ (I) cDNAs that had been labelled with $\left[\alpha^{32} \mathrm{P}\right] \mathrm{dCTP}$ using Rediprime DNA Labelling System, and were autoradiographed on Kodak XAR5 X-ray films.

\section{Statistical Analysis}

Data presented as bar graphs are the means $( \pm$ s.d. $)$ of three to five independent experimental series. Statistical analysis was performed by Student's $t$-test at a $P<0.05$ significance level. 


\section{Results}

\section{Cygb/STAP Expression in Normal Rat Tissues}

Normal rat tissues of the lung, heart, spleen, liver, stomach, small intestine, and kidney were positive in Western blots for Cygb/STAP, whereas those of skeletal muscle and testis were scarce (Figure 1a). Cygb/STAP-expressing cells were immunohistochemically identified on sections of these viscera, skeletal muscle, and skin (Figure 1b). In the kidney, Cygb/STAP expression was dominant in the stromal cells along the proximal and distal uriniferous tubules (Figure 1b-1). The Cygb/STAP-positive cortical stromal cells were also positive for CD73 (ecto-5'-nucleotidase; Figure 1b-2), a marker of renal cortical interstitial fibroblast-like cells. ${ }^{20-22}$ The Cygb/STAP-positive cortical and medullary stromal cells were also positive for HSP47 (Figure 1b-3), a collagen-specific molecular chaperone, ${ }^{23,24}$ but negative for ED1 (Figure 1b-4), a marker of macrophages, ${ }^{13,25}$ and for OX62 (Figure 1b-5), a marker of dendritic cells. ${ }^{11,12}$ Immunoelectron microscopy localized Cygb/STAP-positive immunoprecipitates in the cytoplasm of fibroblast-like cells between the tubules (Figure 1b-6). As shown in Figure 1b-7, Cygb/STAP-positive glomerular cells were positive for Thy1, a marker of mesangial cells. ${ }^{26-28} \mathrm{Cygb} /$ STAP was present in the stromal cells of red pulps in the spleen (Figure 1b-8). These cells were starshaped (Figure 1b-8, inset) and were identical to reticular cells (Figure 1b-9). Smooth muscle cells in the splenic trabecula were negative for Cygb/STAP (data not shown). As in the kidney Cygb/STAPpositive stromal cells were positive for HSP47, but negative for ED1 and OX62 (data not shown). Cygb/ STAP-positive cells were distributed around acini of the pancreas (Figure 1b-10) and were identified with pancreatic stellate cells by immunoelectron microscopy (Figure 1b-11). The stromal cells around interlobular ducts were also Cygb/STAP positive (Figure 1b-10).

The small intestine contained Cygb/STAP-positive stromal cells in the lamina propria, submucosa, and tunica muscularis (Figure 1b-12). They were also positive for HSP47, but negative for ED1 and OX62 (data not shown), and for $\alpha$ SMA (Figure 1b13), a marker of smooth muscle cells. ${ }^{29}$ Cygb/STAP is not present in the interstitial cells of Cajal ${ }^{30}$ as shown by the serial specimens stained by either anti-Cygb/STAP (Figure 1b-14) or anti-c-kit antibodies (Figure 1b-15). Cygb/STAP-positive cells were observed in the lamina propria, submucosa, and tunica muscularis in the stomach and the colon as in the small intestine (data not shown). In the lung, Cygb/STAP-positive cells were present in interstitial tissues along the alveolar walls (Figure 1b-16), and were also found around bronchioles and vessels, but not in the vascular smooth muscle cells (Figure 1b16). In the heart, spindle-shaped cells among cardiac muscle cells were positive for Cygb/STAP (Figure 1b-17), whereas the cardiac muscle cells were negative. Cygb/STAP positivity was also found around capillaries (data not shown). Cygb/STAPpositive spindle-shaped cells were scattered among muscle cells, dominantly around capillaries (Figure 1b-18), in the skeletal tissue, whereas the skeletal muscle cells were negative. In the skin, the epidermis did not contain Cygb/STAP-positive cells (data not shown). Fibroblasts in the dermis were HSP47 positive, but negative for Cygb/STAP (Figure 1b-19). In the dermis Cygb/STAP positivity was observed only around capillaries (data not shown).

\section{Cygb/STAP Expression in Diseased Rat Tissues}

Male WBN/Kob rats are known to suffer from spontaneous pancreatitis. ${ }^{31,32}$ In fact, 20-week-old WBN/Kob rats showed degeneration and necrosis of acinar cells, proliferation of pancreatic ductules, inflammatory cell infiltration, and fibrosis (Figure 2a-1). Cygb/STAP-positive cells were detected in normal regions of acini. In addition, numerous Cygb/STAP-positive spindle-shaped cells were observed in and around fibrotic areas (Figure 2a-2). Double immunostaining showed that these cells were also positive for HSP47 (Figure 2a-3). A part of the Cygb/STAP-positive spindle-shaped cells in the fibrotic area were positive for $\alpha \mathrm{SMA}$, a marker of activated pancreatic stellate cells or myofibroblasts (Figure 2a-4). The Cygb/STAP-positive cells were negative for ED-1 (Figure 2a-5) and OX62 (Figure 2a6). Western blot analysis showed that the diseased pancreas markedly enhanced the expression of Cygb/STAP as well as $\alpha \mathrm{SMA}$ as compared to the normal one (Figure 2a-7).

We repeatedly treated rats under a low-salt $\operatorname{diet}^{10}$ with cyclosporin A, which induced the chronic injury (nephropathy) in uriniferous tubules. Proximal tubules showed degeneration and/or necrosis, and mild fibrosis (Figure 2b-1). Cygb/STAP-positive stromal cells were assembled in the degenerative and/or necrotic tissues (Figure 2b-2). Double immunostaining showed that these cells were also positive for CD73 (Figure 2b-3) and $\alpha$ SMA (Figure 2b-4). These cells were negative for ED-1 (Figure 2b5) and OX62 (Figure 2b-6). Western blot analysis demonstrated that the diseased kidney much elevated the expression of Cygb/STAP as well as $\alpha \mathrm{SMA}$ as compared to the normal one (Figure 2b-7).

\section{Regulation of Cygb/STAP Expression in Primary Cultures of HSCs}

We investigated the factors that regulate the expression of Cygb/STAP in fibroblast-like cell lineage using primary cultures of HSCs as a model because HSCs are the best-characterized vitamin-A storing cell in the splanchnic organs, and their isolation and culture procedures have been well established. ${ }^{5}$ aSMA was induced in HSCs during culture in a time-dependent manner (Figure 3a), a process called 
a

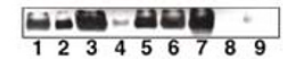

b

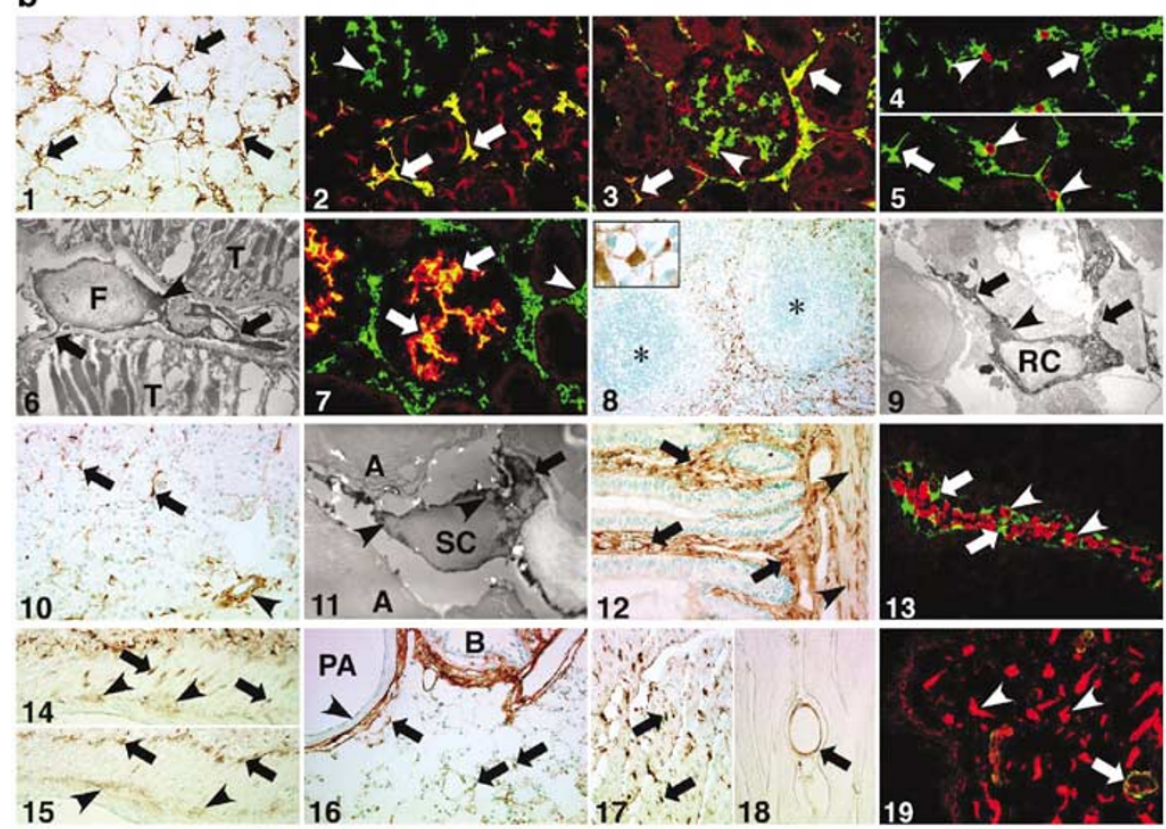

Figure 1 Cygb/STAP expression in normal rat tissues. (a). Western blots of Cygb/STAP in organs. Lane 1, lung; 2, heart; 3, spleen; 4, liver; 5, stomach; 6, small intestine; 7, kidney; 8, skeletal muscle; and 9, testis. (b). Immunohistochemistry of Cygb/STAP on organs. 1-7, kidney. 1. The stromal cells along the uriniferous tubules are positive (arrows). Cygb/STAP-positive cells are also seen in the glomerulus (arrowhead), $\times 130.2$. Double staining of Cygb/STAP (green) and CD73 (red). There are double-positive stromal cells (yellow) along the tubules (arrows). The glomerulus contains single-positive cells for Cygb/STAP (arrowhead), $\times 270$. 3. Double staining of Cygb/STAP (green) and HSP47 (red). There are double-positive stromal cells (yellow) for Cygb/STAP and HSP47 along the tubules (arrows) and in the glomerulus (arrowhead), $\times 270.4$. Fluorescent microscopy for Cygb/STAP (green) and ED1 (red). Cygb/STAP-positive stromal cells (arrow) are clearly distinguishable from ED1-positive cells (arrowhead), $\times 270.5$. Fluorescent microscopy for Cygb/STAP (green) and OX62 (red). Cygb/STAP-positive stromal cells (arrow) obviously differ from the OX62-positive cells (arrowheads), $\times 270$. 6 . Immunoelectron microscopy for Cygb/STAP. Immunoprecipitates are found in the cytoplasm of a fibroblast-like cell (F) between the tubules (T). This cell has fine processes (arrows) and a small lipid droplet (arrowhead), × 5,100. 7. Double staining of Cygb/STAP (green) and Thy1 (red). There are double-positive cells (yellow) in the glomerulus. Single-positive cells for Cygb/STAP are found along the tubules (arrowhead), $\times 270.8$ and 9, spleen. 8. Cygb/STAP immunohistochemistry. Cygb/STAP-positive cells dominate the red pulps and very few in the white pulps (asterisks) and marginal zones, $\times 65$. Inset is a high magnification of a Cygb/STAP-positive and starshaped stromal cell in the splenic code. This cell has fine processes, $\times 800$. 9. Immunoelectron microscopy. A reticular cell (RC) is Cygb/ STAP positive in the cytoplasm and extends some processes (arrows) in the splenic code. This cell has a small lipid droplet (arrowhead), $\times$ 4,100. 10 and 11, pancreas. 10. Cygb/STAP immunohistochemistry. Cygb/STAP-positive spindle-shaped cells are found around acini (arrows). The stromal cells around the interlobular duct are also positive (arrowhead), $\times 130$. 11. Cygb/STAP-immunoelectron microscopy. A stellate cell (SC) around the acinar cells (A) is positive in the cytoplasm. This cell has small lipid droplets (arrowheads) and extends a process $($ arrow) $, \times 4,000.12-15$, small intestine. 12. Cygb/STAP immunohistochemistry. Positive stromal cells are seen in the lamina propria and submucosa (arrows). Spindle-shaped cells in the tunica muscularis are positive (arrowheads), while smooth muscle cells are negative, $\times 130.13$. Fluorescent microscopy for Cygb/STAP (green) and $\alpha$ SMA (red). Stromal cells (arrows) are Cygb/ STAP-positive and evidently distinguished from $\alpha$ SMA-positive cells (arrowheads), $\times 270.14$ and 15 . Serial specimens are stained by anti-Cygb/STAP (14) or anti-c-kit (15) antibodies. Spindle-shaped cells (arrows in 14) in the tunica muscularis are Cygb/STAP positive, while c-kit-positive cells (arrows in 15) are detected between the inner thin and outer thick sublayers of the circular smooth muscle. They show different distributions. Both Cygb/STAP-positive cells (arrowheads in 14) and c-kit-positive ones (arrowheads in 15) are also detected between the circular and longitudinal muscle layers, whereas they obviously show different distributions, $\times 130$. 16. Cygb/ STAP immunohistochemistry on the lung. Stromal cells along alveolar walls (arrows) are positive. Cells around the bronchiole $(B)$ and pulmonary artery $(P A)$ are also positive, while vascular smooth muscle cells (an arrowhead) are negative, $\times 130.17$. Cygb/STAP immunohistochemistry on the heart. Spindle-shaped stromal cells among cardiac muscle cells (arrows) are positive, while cardiac muscle cells are negative, $\times 130$. 18. Cygb/STAP immunohistochemistry on the skeletal muscle. Cygb/STAP was predominant in the stromal cells around a capillary (arrow), while muscle cells are negative for Cygb/STAP, $\times 130$. 19. Fluorescent microscopy for Cygb/ STAP (green) and HSP47 (red) on the dermis. Spindle-shaped cells (arrowheads) are HSP47 positive, but Cygb/STAP negative. Cells around capillaries are Cygb/STAP positive (arrow), $\times 270$.

activation or myofibroblastic transdifferentiation of HSCs. ${ }^{5}$ PDGFR $\beta$, another activation marker of HSCs, was transiently upregulated in the cells (Figure 3a). Cygb/STAP was time-dependently induced during the culture (Figure 3a) in accordance with the results of previous report. ${ }^{1}$ Upregulation of Cygb/ STAP was also observed in the absence of sera, although its level was significantly attenunated. 
PDGFR $\beta$ and $\alpha$ SMA did not show such attenuation (Figure 3a). Sera contain PDGF and TGF $\beta 1$ that accelerate the HSC activation. ${ }^{33}$ Figure $3 \mathrm{~b}$ shows that the Cygb/STAP expression in HSCs was slightly increased by either PDGF-BB or TGF $\beta 1$ in both the presence and absence of serum, suggesting that these factors in the sera may contribute to the induction of Cygb/STAP in HSCs. To know which intracellular signalling is responsible for the induction of Cygb/STAP expression in HSCs, the Cygb/ STAP expression was determined for HSCs stimulated with serum in the presence of PD98059, an MEK inhibitor, LY294002, a PI3 kinase inhibitor, or GF109203, a PKC inhibitor at doses indicated in Figure 3c. Among the three inhibitors tested, only GF109203 suppressed the expression dose-dependently, strongly suggesting that Cygb/STAP expression is activated by the sera through a PKCdependent signaling.

a

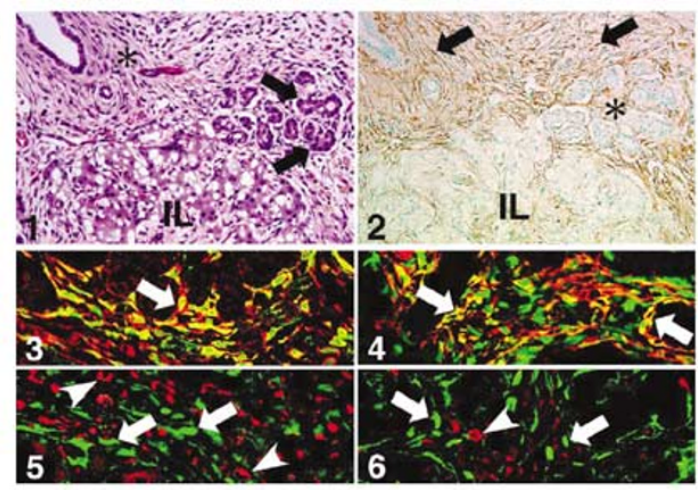

7

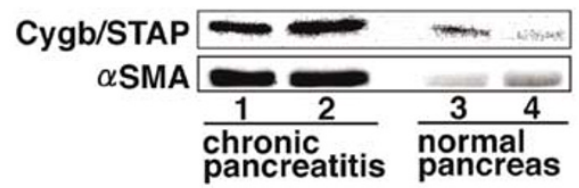

b

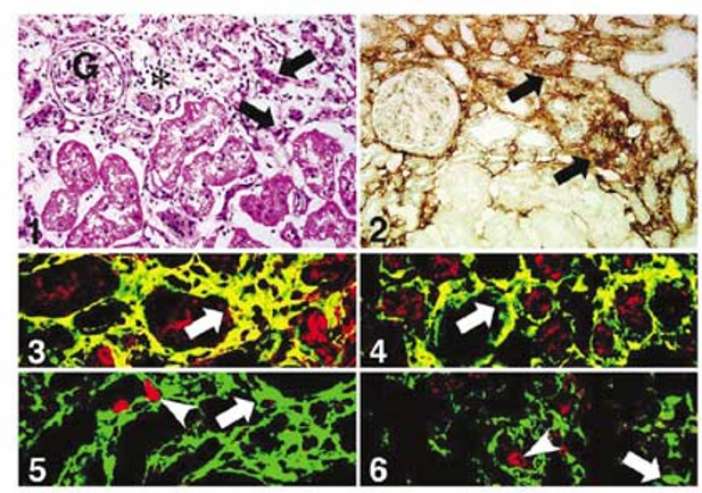

7

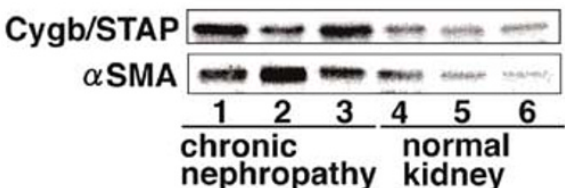

Migration Activity and Collagen mRNA Expression of NIH 3T3 Cells Expressing Rat Cygb/STAP Protein

To investigate the role of Cygb/STAP protein in the process of organ fibrogenesis, we generated F9 cells, an NIH 3T3 cell line transfected with rat Cygb/STAP cDNA, and observed their morphology and migratory potential. Cygb/STAP protein was not detectable in wild-type or pZeoSV2/lacZ-transfected NIH 3T3 cells (LacZ cells) (Figure 4A). In contrast, F9 cells exhibited detectable Cygb/STAP in Western blots (Figure 4A) and also in indirect immunocytochemistry (data not shown) that showed the ubiquitous localization of the protein in the cytoplasm. Wild-type and LacZ cells showed a locomoting phenotype with membrane rufflings (Figure 4Ba) and randomly distributed vinculin in their cytoplasm (Figure 4B-c). In contrast, F9 cells exhibited flattened and stretched shapes with developed F-actin stress fibres (Figure 4B-b) and accumulated vinculin at their focal contacts (Figure 4B-d). These observations suggested that F9 cells attached tightly to substrata, which in turn might hamper the locomotion of the cells. In fact, F9 cells

Figure $2 \mathrm{Cygb} / \mathrm{STAP}$ expression in diseased rat tissues. (a) The chronic pancreatitis of 20 -week-old WBN/Kob rats. 1. HEstaining. Fibrotic areas (asterisk) are extended around the degenerate acini (arrows). IL, islets of Langerhans, $\times 130.2$. Immunostaining for Cygb/STAP. Fibrotic areas around the acini (asterisk) are composed of numerous Cygb/STAP-positive spindle-shaped stromal cells (arrows). IL, islets of Langerhans, $\times 130$. 3. Double immunostaining for Cygb/STAP (green) and HSP47 $(r e d)$. Stromal cells in the fibrotic area and around the acini (arrow) are double positive (yellow), $\times 270.4$. Double staining of Cygb/STAP (green) and $\alpha$ SMA (red). Some of the spindle-shaped cells in the fibrotic area (arrows) are double positive (yellow), $\times 270$. 5. Fluorescent microscopy for Cygb/STAP (green) and ED1 (red). Cygb/STAP-positive stromal cells (arrows) are evidently distinguished from ED1-positive cells (arowheads), $\times 270.6$. Fluorescent microscopy for Cygb/STAP (green) and OX62 (red). Cygb/STAP-positive stromal cells (arrows) are obviously different from OX62-positive cells (arrowhead), $\times 270$. 7. Western blot analysis of Cygb/STAP and $\alpha \mathrm{SMA}$ in the pancreas of WBN/Kob rats and normal Wistar rats. Lanes 1 and 2, WBN/Kob rats; Lanes 3 and 4, normal Wistar rats. (b) The kidney of cyclosporin Ainduced chronic nephropathy. 1. HE staining. There is degeneration and/or necrosis in proximal uriniferous tubules (arrows) and mild fibrosis in the cortex (an asterisk). G, glomerulus, $\times 130.2$. Immunostaining for Cygb/STAP. Cygb/STAP-positive stromal cells are accumulated in the necrotic area of tubules (arrows), $\times 130$. 3. Double staining of Cygb/STAP (green) and CD73 (red). There are double-positive stromal cells (yellow) in the necrotic area of tubules (arrow), $\times 270.4$. Double staining of Cygb/STAP (green) and $\alpha \mathrm{SMA}$ (red). There are double-positive spindleshaped cells (yellow) in the necrotic area of tubules (arrow), $\times$ 270. 5. Fluorescent microscopy for Cygb/STAP (green) and ED1 (red). Cygb/STAP-positive stromal cells (arrow) are evidently distinguished from ED1-positive cells (arowhead), $\times 270$. 6 . Fluorescent microscopy for Cygb/STAP (green) and OX62 (red). Cygb/STAP-positive stromal cells (arrow) are obviously different from OX62-positive cells (arrowhead), $\times 270$. 7. Western blot analysis of Cygb/STAP and $\alpha$ SMA in the kidney of cyclosporin Ainduced chronic nephropathy rats and nontreated control rats. Lanes 1-3, cyclosporin A-induced chronic nephropathy rats; Lanes 4-6, normal SD rats. 

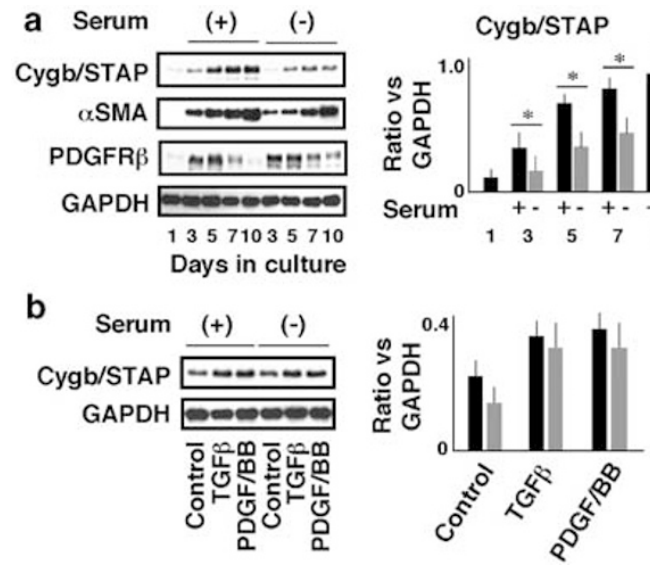

aSMA

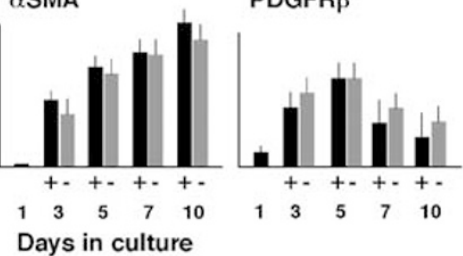

b

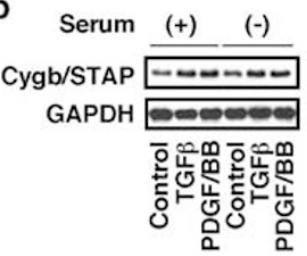

C

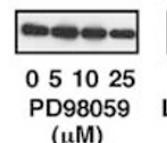

$(\mu \mathrm{M})$

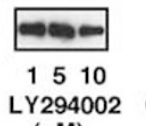

$(\mu \mathrm{M})$

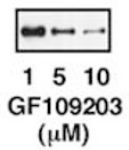

Figure 3 Regulation of Cygb/STAP expression in primary cultures of rat HSCs. (a) Expression of Cygb/STAP, $\alpha$ SMA, and PDGFR $\beta$ in HSCs. Cells were cultured for $24 \mathrm{~h}$ in DMEM containing 10\% FBS and were additionally cultured with or without $10 \%$ FBS for up to 10 days. The amount of Cygb/STAP, $\alpha$ SMA, and PDGFR $\beta$ in cells of each experiment was determined by Western blotting. The expression of glyceraldehyde-3-phosphate dehydrogenase (GAPDH) in HSCs was used as an internal standard. The intensities of visualized bands were scanned by Imaging Densitometer GS-700 (BIO-RAD, Hercules, CA, USA). Bars depict mean \pm s.d. of three individual experiments. ${ }^{*} P<0.05$. Black and grey bars represent the value in the presence and absence of serum, respectively. (b) Expression of Cygb/STAP in HSCs. HSCs were cultured for $24 \mathrm{~h}$ as in a and additionally cultured for $48 \mathrm{~h}$ with or without FBS supplemented with either $20 \mathrm{ng} / \mathrm{ml}$ rat recombinant PDGF-BB or $100 \mathrm{ng} / \mathrm{ml}$ human recombinant TGF $\beta 1$. The expression level of Cygb/STAP was determined by Western blotting. The expression of GAPDH was used as an internal standard. The intensities of visualized bands were scanned by Imaging Densitometer GS-700 (BIO-RAD, Hercules, CA, USA). Bars depict mean \pm s.d. of three individual experiments. Black and grey bars represent the value in the presence and absence of serum, respectively. (c) Cygb/STAP expression in the presence of protein kinase inhibitors. HSCs were cultured for $24 \mathrm{~h}$ as in (a) and additionally cultured for $48 \mathrm{~h}$ with $10 \%$ FBS supplemented with either PD98059, LY294002, or GF109203 at the indicated dose. The expression level of Cygb/STAP was determined by Western blotting.

showed siginificantly less migration activity as compared to wild-type and LacZ cells (Figure 4c).

Three types of NIH 3T3 cells, wild-type, LacZ cells, and F9 cells, were cultured in serum-free media for $24 \mathrm{~h}$ in the absence or presence of $100 \mathrm{ng} /$ $\mathrm{ml}$ of TGF- $\beta 1$. Collagen $\alpha 1$ (I) mRNA expression was determined by Northern blotting for each of them (Figure 5). TGF- $\beta$ slightly stimulated collegen $\alpha 1$ (I) mRNA expression in wild-type and LacZ cells and drastically augmented it in F9 cells, which suggested a role of Cygb/STAP protein as a stimulator in TGF- $\beta$-dependent collagen synthesis.

\section{Discussion}

In the present study, we performed immunohistochemistry, Western blotting, and immunoelectorn microscopy on various visceral organs to localize and identify Cygb/STAP-expressing cells. As a result, we could demonstrate that a variety of visceral organs contain Cygb/STAP-positive cells in addition to liver where Cygb/STAP was originally described in relation to HSC activation. ${ }^{1}$ These positive cells were star- or spindle-shaped, and existed in the parenchyma and interlobular connective tissues. They were obviously distinguished from any epithelial cells and blood cells. Smooth, vascular smooth, cardiac, and skeletal muscle cells were all Cygb/STAP negative. Double immunostaining showed that Cygb/STAP-positive cells were negative for ED1 and OX-62, a marker of macrophages $^{13,25}$ and dendritic cells, ${ }^{11,12}$ respectively.
Double immunostaining also showed that Cygb/ STAP-positive cells were positive for HSP47, strongly suggesting that the cells may produce collagens. ${ }^{23,24}$ Taken together, we conclude that Cygb/STAP-positive cells are splanchnic fibroblastlike cells. Interestingly, the dermis also contains Cygb/STAP-positive cells, but these cells are not fibroblasts. The positive cells were observed only in pericytes around capillaries. HSP47-positive cells in the dermis were negative in Cygb/STAP staining. Since these HSP47-positive cells are considered to be skin fibroblasts, it is said that dermal fibroblasts do not express Cygb/STAP.

Cygb/STAP was discovered by a proteome analysis for the activation of rat HSCs. ${ }^{1,34}$ HSCs are liverspecific fibroblast-like cells characterized by the presence of vitamin A-containing lipid droplets. ${ }^{8}$ Furthermore, various splanchnic organs besides liver possess fibroblast-like cells storing vitamin A. ${ }^{8}$ For instance, it was recently reported that there are fibroblast-like cells with lipid droplets in the periacinar region of pancreas. ${ }^{35,36}$ These cells were named the pancreatic stellate cells. In the kidney, vitamin A-positive cells were found in the area between the tubules. ${ }^{37}$ In the spleen, vitamin A positivity was observed in the reticular cells in the splenic codes of the red pulps. ${ }^{8,38}$ In addition, vitamin A-storing cells were distributed throughout the alimentary canal and were seen in the lamina propria, especially in the villi, submucosal layer, and adventitia. ${ }^{8}$ Yamada and Hirosawa ${ }^{7}$ proposed a common classification for these vitamin A-storing cells under the name 'vitamin A-storing cell system'. 
A
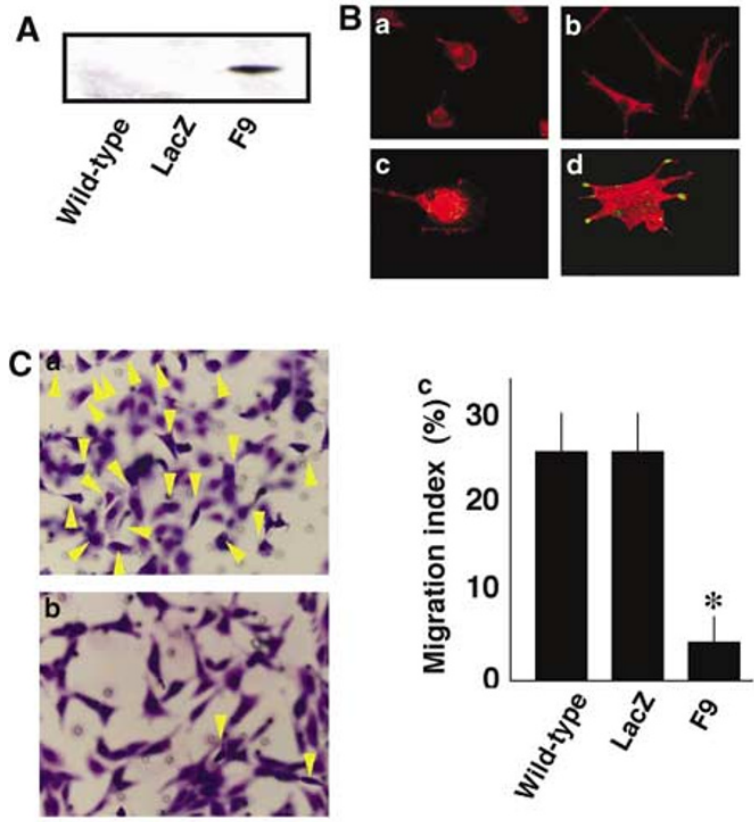

Figure 4 Effects of Cygb/STAP expression on the morphology and locomotion activity of NIH 3T3 cells. (A) Western blot analysis of Cygb/STAP expression in NIH 3T3 cells. NIH 3T3 cells were transfected with either pZeoSV2/lacZ (LacZ) or pZeoSV2/ Cygb/ STAP (F9). Cell lysates $(10 \mu \mathrm{g})$ were prepared and used for Western blotting. (B) Morphology of NIH3T3 cells. LacZ cells (a and $\mathbf{c}$ ) or F9 cells (b and $\mathbf{d}$ ) were placed in $35 \mathrm{~mm}$-cell culture plates and incubated overnight. The cells were fixed and stained for F-actin (a and b) or double stained for F-actin (red) and vinculin (green, $\mathbf{c}$ and $\mathbf{d}$ ). The specimens were observed under a fluorescent microscope. (C) Migration activity of NIH 3T3 cells. Cell migration was assayed using Cell Culture Insert. Cells $\left(10^{5}\right.$ cells) were detached from the plates using trypsin, suspended in $400 \mu \mathrm{l}$ of $10 \% \mathrm{FBS} / \mathrm{DMEM}$, introduced into the insert, and allowed to adhere to the upper surface of the membrane. The cells were then maintained in 10\% FBS/DMEM for $24 \mathrm{~h}$. The culture media were removed. Cells adhering to the membrane were fixed by $100 \%$ methanol and stained with a Giemsa solution. Representative pictures of the migration are shown for LacZ cells (a) and F9 cells (b) at a $\times 400$ magnification. Arrow heads indicate the cells that migrated to the lower surface of the culture insert through pores. The number of cells on the upper surface of the membrane was counted and similarly for the lower surface by changing the focus. Five microscopic fields were randomly chosen for each specimen. MI was calculated (c). F9 cells show a significantly low migration potential. ${ }^{*} P<0.01$ as compared to wild-type and LacZ cells.

The present study confirmed that all these splanchnic vitamin A-storing cells express Cygb/STAP in normal conditions.

On the other hand, Yamamoto et al ${ }^{39}$ reported that vitamin A fluorescence was seen in the fibroblasts of the dermis after the administration of excess vitamin A. Since the present study shows that fibroblasts in the dermis are negative for Cygb/STAP, it can be said that there is a difference between splanchnic fibroblasts and dermis fibroblasts in the 'vitamin A-storing cell system'. Wake ${ }^{40,41}$ proposed a suggestion to solve this problem in the study using the lamprey, Lampetra japonica. Lampreys are well known to contain a large amount of vitamin $\mathrm{A}$ in
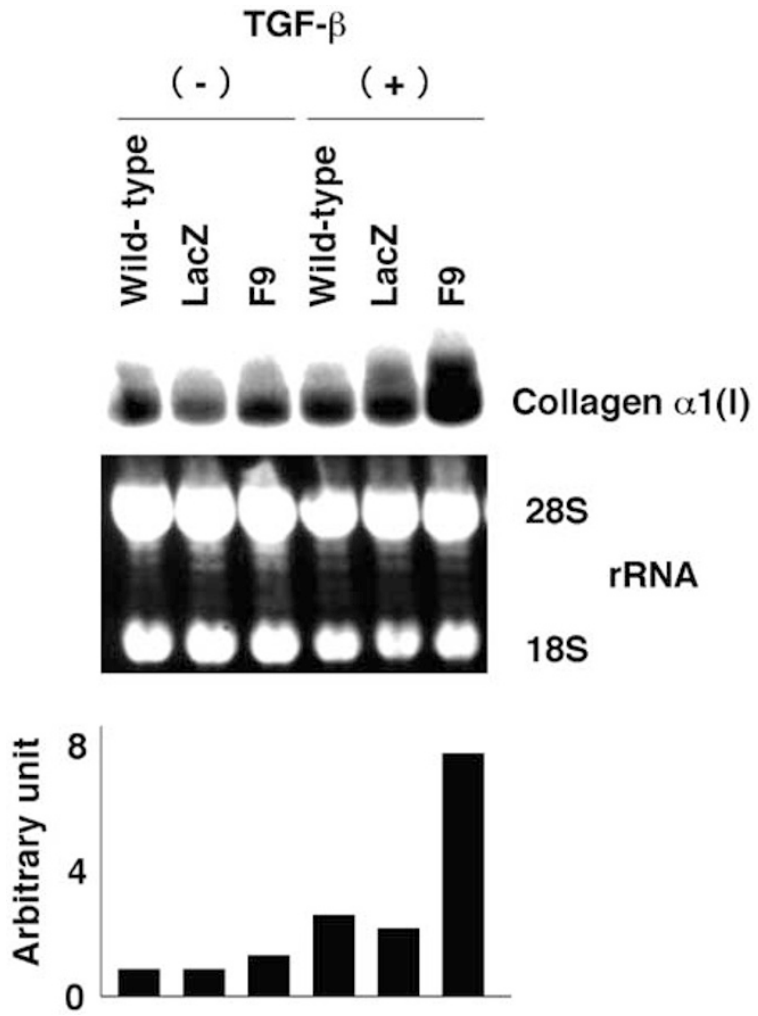

Figure 5 Effects of Cygb/STAP expression on collagen $\alpha 1$ (I) mRNA in NIH 3T3 cells. Three types of NIH 3T3 cells, wildtype, LacZ cells, and F9 cells, were cultured in serum-free media for $24 \mathrm{~h}$ in the absence or presence of $100 \mathrm{ng} / \mathrm{ml}$ of TGF- $\beta 1$. The expression of collagen $\alpha 1$ (I) mRNA was determined by Northern blotting for each of them. Bar graphs indicate the mean of the relative intensity of each band from two individual experiments against the intensity of the band of wild-type NIH 3T3 cells.

their bodies. By using Kupffer's gold chloride method, numerous vitamin A-storing cells were shown to be distributed in their various splanchnic organs, such as the liver, digestive canal, kidney, gill, pancreas, and heart. In the kidney, vitamin A reaction was seen in the mesangial cells. ${ }^{42}$ On the contrary, vitamin A-storing cells were scarce or absent in the dermis, subcutaneous connective tissues, and connective tissues around the notochord. Thus, there might exist two fibroblastic cell lineages: (1) conventional fibroblasts, which originate from the mesenchyme of dorsal mesoderm and somatic mesoderm of lateral mesoderm, and (2) vitamin A-storing fibroblast-like cells, which are derived from the mesenchyme of intermediate mesoderm and splanchnic mesoderm of lateral mesoderm. ${ }^{40,41}$ In this context, the present study proposes that Cygb/STAP is a novel and useful marker for splanchnic vitamin A-storing fibroblastlike cells.

We investigated Cygb/STAP expression in two diseased fibrotic models. In the $\mathrm{WBN} / \mathrm{Kob}$ rats developing chronic spontaneous pancreatitis, ${ }^{31,32}$ numerous Cygb/STAP-positive cells appeared in the fibrotic tissues. These cells were regarded as 
fibroblast-like cells by their morphological features and results of double-immunostaining analysis for anti-HSP47 and anti-ED1 or OX62 antibodies. The pancreatic stellate cells around acini are activated during fibrosis in a manner similar to that of HSCs in the liver during fibrosis. ${ }^{43}$ In fact, Cygb/STAPpositive fibroblast-like cells in the fibrotic area coexpressed $\alpha$ SMA, a marker of activated pancreatic stellate cells. ${ }^{43}$ Thus, these Cygb/STAP-positive fibroblast-like cells are considered as activated or myofibroblastic pancreatic stellate cells.

In the cyclosporin A-induced rat chronic nephropathy, Cygb/STAP-positive and star-shaped interstitial cells were observed in degenerated or necrotic areas of uriniferous tubules. These cells were considered as fibroblast-like cells by their morphological features and the results of double-immunostaining analysis using anti-CD73 and anti-ED1 or OX62 antibodies. Since these interstitial cells were also positive for $\alpha \mathrm{SMA}$, they were suggested to be activated and myofibroblastic cells. ${ }^{44-46}$

The function of Cygb/STAP in normal and diseased rat tissues is still unknown. The present study strongly suggests a role of Cygb/STAP in fibrogenesis, because, firstly, Cygb/STAP is expressed in vitamin A-storing HSP47-positive splanchnic fibroblast-like cell lineages and, secondly, Cygb/STAP expression is induced in chronically damaged tissues. In fact, Cygb/STAP is induced in HSCs, the major cell source to produce collagen and other extracellular matrices in the injured liver, in response to sera, and fibrotic growth factors, such as PDGF-BB and TGF- $\beta$. Cygb/STAP has been shown to be an $\mathrm{O}_{2}$-binding globin as haemoglobin, myoglobin, and Ngb. ${ }^{1-4}$ However, there has been no explanatory relation among the $\mathrm{O}_{2}$-binding, vitamin A metabolism, and collagen synthesis. Cygb/STAP may play a role as an oxygen sensor and might be a stress-sensitive molecule because Cygb/STAP is specifically localized around capillaries where hypoxic conditions affect cellular functions.

During the preparation of this paper, Geuens et al ${ }^{47}$ reported the expression of Cygb in mouse brain and liver. Cygb expression in the liver is dominant in hepatocytes and their nuclei, which contradicts our previous report using rats that Cygb/STAP is localized in the cytoplasm of HSCs, but not in the nuclei of hepatocytes, ${ }^{1}$ and the present study that Cygb/STAP is absent from any epithelial cells. Although we have no explanation for this discrepancy presently, difference in animal species and polyclonal antibodies used in individual experiments might induce such results. There is the possibility that Cygb/STAP could function as a nuclear protein as well, because we observed that Cygb/STAP was also immunocytochemically positive in the nuclei of some of F9 cells (data not shown).

Our analysis using NIH3T3 cells showed that Cygb/STAP overexpression induced the formation of actin stress fibres and focal contact and, as a result, it impared significantly cell migration. We speculate that the activation of Rho may be involved because, firstly, Rho activation is essential for actin stress fibre formation ${ }^{48,49}$ and, secondly, constitutive activation of Rho signalling inhibited cell migration in NIH3T3 cells ${ }^{50}$ and vascular endothelial cells. ${ }^{51}$ This speculation is supported by our observation showing that Y27632, a specific Rho-associated coiled-coil-forming protein serine/ threonine kinase inhibitor, inhibited the formation of actin stress fibres in F9 cells (data not shown). In addition, we hardly detected the enhanced cell proliferation and activation of extracellular signal-regulated kinase $1 / 2$ and Akt under the stimulation with either serum or platelet-derived growth factor-BB in F9 cells (data not shown). It is thus plausible that integlinand focal adhesion kinase-dependent signal pathways may not be augmented in F9 cells. Taking these data obtained by Cygb/STAP-expressing NIH3T3 cells into account, it can be said that Cygb/STAP triggers the transformation of fibroblasts to myofibroblastic cells that express well-developed actin stress fibres and stimulates collagen production at the inflammatory sites. Collagen $\alpha 1$ (I) mRNA expression of F9 cells was markedly enhanced in the presence of TGF- $\beta$. Thus, it seems that Cygb/STAP protein plays a role as a stimulator of collagen mRNA transcription. This role is in agreement with the possible involvement of Cygb/STAP in the process of organ fibrosis disscussed above.

In conclusion, the present study showed that Cygb/STAP is expressed in the splanchnic fibroblast-like cells of various normal visceral organs, which places Cygb/STAP in a position as a useful new marker of splanchnic 'vitamin A-storing' fibroblast cell species. Nectrotic or fibrotic interstitial tissues of diseased organs such as the kidney and pancreas accumulated strongly Cygb/STAPpositive fibroblast-like cells. Cytoglobin/STAP overexpression induced fibroblasts to lessen migratory activities and increase collagen $\alpha 1(\mathrm{I})$ mRNA expression. Thus, it can be said that Cygb/STAP is a tissue globin uniquely localized in splanchnic fibroblastic cell lineage and may play a role in fibrotic organ disorder.

\section{Acknowledgement}

We thank Professor Kenjiro Wake, Minophagen Pharmaceutical Co. (Tokyo, Japan), and Dr Kenji Kaneda, Houai Hospital (Osaka, Japan) for the valuable comments on this work. We also thank Drs Mitsue Sogawa and Masaki Hamaguchi, Osaka City University for valuable technical support. This work was supported in part by Grant-in-aid from the Ministry of Education, Science and Culture of Japan (14570497) and by Ono Medical Research Foundation. 


\section{References}

1 Kawada N, Kristensen DB, Asahina K, et al . Characterization of a stellate cell activation-associated protein (STAP) with peroxidase activity found in rat hepatic stellate cells. J Biol Chem 2001;276:2531825323.

2 Burmester T, Ebner B, Weich B, et al. Cytoglobin: a novel globin type ubiquitously expressed in vertebrate tissues. Mol Biol Evol 2002;19:416-421.

3 Pesce A, Bolognesi M, Bocedi A, et al. Neuroglobin and cytoglobin. Fresh blood for the vertebrate globin family. EMBO reports 2002;3:1146-1151.

4 Trent III JT, Hargrove MS. A ubiquitously expressed human hexacoordinate hemoglobin. J Biol Chem 2002;277:19538-19545.

5 Kawada N. The hepatic perisinusoidal stellate cell. Histol Histopathol 1997;12:1069-1080.

6 Asahina K, Kawada N, Kristensen DB, et al. Characterization of human stellate cell activation-associated protein and its expression in human liver. Biochim Biophys Acta 2002;1577:471-475.

7 Yamada E, Hirosawa K. The possible existence of a vitamin A-storing cell system. Cell Struct Funct 1976;1:201-204.

8 Wake K. Perisinusoidal stellate cells (fat-storing cells, interstitial cells, lipocytes), their related structure in and around the liver sinusoids, and vitamin A-storing cells in extrahepatic organs. Int Rev Cytol 1980;66:303-353.

9 Komuro T. Re-evaluation of fibroblasts and fibroblastlike cells. Anat Embryol 1990;182:103-112.

10 Burdmann EA, Andoh TF, Nast CC, et al. Prevention of experimental cyclosporin-induced interstitial fibrosis by losartan and enalapril. Am J Physiol 1995;269:F491-F499.

11 Brenan M, Puklavec M. The MRC OX-62 antigen: a useful marker in the purification of rat veiled cells with the biochemical properties of an integrin. J Exp Med 1992;175:1457-1465.

12 Brissette-Storkus CS, Kettel JC, Whitham TF, et al. Flt3 ligand (FL) drives differentiation of rat bone marrowderived dendritic cells expressing OX62 and/or CD161 (NKR-P1). J Leukocyte Biol 2002;71:941-949.

13 Tao LH, Enzan H, Hayashi Y, et al. Appearance of denuded hepatic stellate cells and their subsequent myofibroblast-like transformation during the early stage of biliary fibrosis in the rat. Med Electron Microsc 2000;33:217-230.

14 Vannucchi MG, Corsani L, Faussone-Pellegrini MS. Substance $\mathrm{P}$ immunoreactive nerves and interstitial cells of Cajal in the rat and guinea-pig ileum. A histochemical and quantitative study. Neurosci Lett 1999;268:49-52.

15 Nishihara E, Nagayama Y, Inoue S, et al. Ontogenetic changes in the expression of estrogen receptor alpha and beta in rat pituitary gland detected by immunohistochemistry. Endocrinology 2000;141:615-620.

16 Nakatani K, Seki S, Kawada N, et al. Expression of SPARC by activated hepatic stellate cells and its correlation with the stages of fibrogenesis in human chronic hepatitis. Virchows Archiv 2002; 441:466-474.

17 Nakatani K, Seki S, Kawada N, et al. Expression of neural cell adhesion molecule (N-CAM) in perisinusoidal stellate cells of the human liver. Cell Tissue Res 1996;283:159-165.
18 Kobayashi S, Seki S, Kawada N, et al. Apoptosis of T cells in the hepatic fibrotic tissue of the rat: a possible inducing role of hepatic myofibroblast-like cells. Cell Tissue Res 2003;311:353-364.

19 Ikeda K, Wakahara T, Wang YQ, et al. In vitro migratory potential of rat quiescent hepatic stellate cells and its augmentation by cell activation. Hepatology 1999;29:1760-1767.

20 Le Hir M, Kaissling B. Distribution of 5'-nucleotidase in the renal interstitium of the rat. Cell Tissue Res 1989;258:177-182.

21 Kaissling B, Hegyi I, Loffing J, et al. Morphology of interstitial cells in the healthy kidney. Anat Embryol 1996;193:303-318.

22 Tisher CC, Madsen KM. Anatomy of the kidney, In: Brenner BM (ed). Brenner and Rector's the Kidney, 6th edn W.B. Saunders: Philadelphia, 2000, 3-67.

23 Kawada N, Kuroki T, Kobayashi K, et al. Expression of heat-shock protein 47 in mouse liver. Cell Tissue Res 1996;284:341-346.

24 Nagata K. Hsp47: a collagen-specific molecular chaperone. Trends Biochem Sci 1996;21:22-26.

25 Sogawa M, Matsumoto T, Yamagami H, et al. A murine model of granulomatous colitis with mesenteric lymphadenitis induced by mycobacterial cord factor. Virchows Arch 2003;442:151-158.

26 Yamamoto $\mathrm{T}$, Yamamoto $\mathrm{K}$, Kawasaki $\mathrm{K}$, et al. Immunoelectron microscopic demonstration of Thy-1 antigen on the surfaces of mesangial cells in the rat glomerulus. Nephron 1986;43:293-298.

27 Yaoita E, Yamamoto T, Takashima N, et al. Visceral epithelial cells in rat glomerular cell culture. Eur J Cell Biol 1995;67:136-144.

28 Ardaillou R, Ronco P, Rondeau E, et al. Biology of renal cells in culture, In: Brenner BM (ed) Brenner and Rector's the Kidney, 6th edn. W.B. Saunders: Philadelphia, 2000, 93-191.

29 Skalli O, Ropraz P, Trzeciak A, et al. A monoclonal antibody against alpha-smooth muscle actin: a new probe for smooth muscle differentiation. J Cell Biol 1986;103:2787-2796.

30 Komuro T, Seki K, Horiguchi K. Ultrastructural characterization of the interstitial cells of Cajal. Arch Histol Cytol 199;62:295-316.

31 Ohashi K, Kim JH, Hara H, et al. WBN/Kob rats. A new spontaneously occurring model of chronic pancreatitis. Int J Pancreatol 1990;6:231-247.

32 Kakinuma C, Suda K, Shibutani Y. Histopathological and biochemical studies on pancreatic fibrosis in WBN/Kob rats. Virchows Arch 1999; 434:83-89.

33 Pinzani M, Marra F. Cytokine receptors and signaling in hepatic stellate cells. Semin Liver Dis 2001;21: 397-416.

34 Kristensen DB, Kawada N, Imamura K, et al. Proteome analysis of rat hepatic stellate cells. Hepatology 2000;32:268-277.

35 Apte MV, Haber PS, Applegate TL, et al. Periacinar stellate shaped cells in rat pancreas: identification, isolation, and culture. Gut 1998;43:128-133.

36 Bachem MG, Schneider E, Gross H, et al. Identification, culture, and characterization of pancreatic stellate cells in rats and humans. Gastroenterology 1998;115:421-432.

37 Nagy NE, Holven KB, Roos N, et al. Storage of vitamin A in extrahepatic stellate cells in normal rats. J Lipid Res 1997;38:645-658. 
38 Kusumoto Y, Fujita T. Vitamin A uptake cells distributed in the liver and other organs of the rat. Arch Histol Jap 1977;40:121-136.

39 Yamamoto M, Enzan H, Hara H, et al. Fluorescence and electron microscopic studies on the perivascular mesenchymal cells and fibroblasts after vitamin A administration. Acta Pathol Jap 1978;28:513-521.

40 Wake K. The sternzellen of von Kuppfer-after 106 years, In: Knook DL, Wisse E, (eds) Sinusoidal Liver Cells. Elsevier Biomedical Press: The Netherlands, 1982, 1-12.

41 Wake K. One hundred years of sinusoidal cells in the liver. Acta Anat Nippon 1997;72:407-423.

42 Bauer P, Wake K. Mesangial cells of the lamprey, Lampetra japonica, store vitamin A. Arch Histol Cytol 1996;59:71-78.

43 Haber PS, Keogh GW, Apte MV, et al. Activation of pancreatic stellate cells in human and experimental pancreatic fibrosis. Am J Pathol 1999;155:1087-1095.

44 Schürch W, Seemayer TA, Gabbiani G. Myofibroblast, In: Sternberg SS (ed). Histology for Pathologists, 2nd edn. Lippincott-Raven Publishers: Philadelphia, 1997, pp 129-165.

45 Badid C, Mounier N, Costa AMA, et al. Role of myofibroblasts during normal tissue repair and excessive scarring: interest of their assessment in nephropathies. Histol Histopathol 2000;15: 269-280.

46 Zeisberg M, Strutz F, Müller GA. Role of fibroblast activation in inducing interstitial fibrosis. J Nephrol 2000;13:S111-S120.

47 Geuens E, Brouns I, Flamez D, et al. A globin in the nucleus. J Biol Chem 2000;278:30417-30420.

48 Ridley AJ, Hall A. The small GTP-binding protein rho regulates the assembly of focal adhesions and actin stress fibers in response to growth factors. Cell 1992;70:389-399.

49 Luttrell LM. Big G, little G: G proteins and actin cytoskeletal reorganization. Mol Cell 2002;9: 1152-1154.

50 Olivo C, Vanni C, Mancini P, et al. Distinct involvement of cdc42 and RhoA GTPases in actin organization and cell shape in untransformed and Dbl oncogene transformed NIH3T3 cells. Oncogene 2000;19 1428-1436.

51 Verma A, Davis GE, Ihler GM. Formation of stress fibres in human endothelial cells infected with Bartonella bacilliformis is associated with altered morphology, impaired migration and defects in cell morphogenesis. Cell Microbiol 2001;3:169-180. 